\title{
Novel Biomarkers for Early Diagnosis and Progression of Diabetic Nephropathy
}

\author{
Li-hua Liu ${ }^{1}$, Qiao-yan Guo ${ }^{1}$, Chao-yuan Li $^{1}$, Wen-peng Dong ${ }^{2}$, Guang-dong Sun ${ }^{1}$ \\ ${ }^{1}$ Department of Nephrology, Second Hospital of Jilin University, Changchun, China \\ ${ }^{2}$ Blood Purification Center, Daqing Oilfield General Hospital,Heilongjiang, China \\ Correspondence should be addressed to Guang-dong Sun, sungd@jlu.edu.cn
}

\begin{abstract}
Diabetic nephropathy $(D N)$ is one of the most common diabetic microvascular complications and is defined as a rise in urine albumin excretion (UAE) rate and progressive renal function loss. Now albuminuria is considered the gold standard of onset or progression of DN. However albuminuria has certain limitations, clinical practice suggested detectable albuminuria is later than the onset of DN and subsequent intervention could not block the progression of DN effectively, which could lead to renal function deterioration influencing morbidity and mortality. Thus, the request for more reliable highly sensitive and specific biomarkers is needed for early predicting the onset and progression of DN, which is important to slow down or prevent the renal function decline in diabetic patients. Tremendous studies have implicated lots of biomarkers associated with DN could be applied to predict the onset or monitor the progression of DN. In this review, we summarize and screen a lot of already known biomarkers, the newly reported biomarkers in urine and serum,such as serum sKloth, Serum angiopoietin-like protein 2 (Angptl2), and urinary vitamin D-binding protein (VDBP), predicting development and progression of DN, which could be potential useful tools in the management of DN.
\end{abstract}

Keywords: diabetic nephropathy, biomarker, early diagnosis, serum, urine

\section{INTRODUCTION}

DN is the most serious microvascular complications of both type 1 and type 2 diabetes, which significantly influenced patient morbidity, mortality and life quality. DN is the leading cause of end stage renal failure (ESRD) in developed and developing countries. The gold standard biomarker of DN is considered to be albuminuria in clinical practice, decrease or increase in urinary albumin levels was defined as remission or progression of DN, while albuminuria has lots of limitations such as low sensitivity and larger variability, it can't predictably lead to a renal outcome nor are specific for DN(Jim et al. 2012). Thus apart from microalbuminuria, earlier, more specific and sensitive biomarkers with greater predictability are needed for early and correct diagnosis and prediction of progression in DN. The focus of this review is to summarize novel biomarkers in urine, serum for renal injury associated with DN, which can be used in the early diagnosis of DN or in the evaluation of the treatment to prevent, slow down or reverse the progression of DN.

\section{URINARY BIOMARKERS FOR DN}

\subsection{Cystatin C}

Cystatin $\mathrm{C}$, a $13 \mathrm{kDa}$ cysteine protease inhibitor, freely filtered by the renal glomeruli, is a novel biomarker of renal failure. Urinary cystatin $\mathrm{C}$ has been associated with the decline of eGFR and predictors of the progression of type 2 DN (Kim et al. 2013). One experimental study in Zucker diabetic fatty (ZDF) rats indicated that urinary cystatin $\mathrm{C}$ levels were increased in ZDF rats while renal damage was not histopathologically observed, and cystatin $\mathrm{C}$ further increased with the progression of renal damage, which demonstrated the usefulness of early detection and accurate assessment of DN(Togashi and Miyamoto 2013). Urinary cystatin C level was identified as independent factor associated with eGFR less than $60 \mathrm{ml} / \mathrm{min} / 1.73 \mathrm{~m}^{2}$ in type 2 diabetic patients with 


\section{Li-Hualiu et al.}

normoalbuminuria, which suggested that cystatin $\mathrm{C}$ could be useful biomarker for the early detection of DN(Jeon et al. 2011). Furthermore, cystatin $\mathrm{C}$ with other low-molecular-weight proteins filtered then entirely reabsorbed in the proximal tubule should be noted,urinary cystatin $\mathrm{C}$ increases independent of serum cystatin $\mathrm{C}$ suggesting renal tubular damage rather than solely glomerular damage in DN(Lee and Choi 2014).

\section{IMMUNOGLOBULINS}

Plasma cells can secret immunoglobulins including $\mathrm{M}$ and $\mathrm{G}$, which can excrete in urine in DN patients.

Immunoglobulin $\mathrm{M}(\operatorname{IgM})$ is the largest antibody in human, urinary $\operatorname{IgM}$ excretion indicates that a large, nonselective pore damage in the glomerular capillary wall due to IgM large molecular radius. An early study showed that patients with type 2 DN and with nephronsclerosis had significantly higher urinary IgM excretion compared to the age-matched healthy controls(Bakoush et al. 2002). Another study showed that renal survival of type $1 \mathrm{DN}$ patients were inversely associated with urinary IgM excretion, which indicated that increased urinary IgM excretion was an early predictor of type 1 DN progression than albuminuria. A recent study showed that the risk of renal failure was 4.9 fold higher in patients with increased urinary IgM excretion compared to patients with low urinary $\operatorname{IgM}$ excretion, which indicated that an increased urinary $\operatorname{IgM}$ excretion was an independent predictor of renal death irrespective of the degree of albuminuria(Tofik et al. 2012).

Immunoglobulin $\mathrm{G}(\mathrm{IgG})$ is a plasma protein larger than albumin (MW $150 \mathrm{kDa}$ ). A systemic review indicated that urinary $\mathrm{IgG}$ excretion could be a good marker for predicting the onset of DN (Hellemons et al. 2012). Urinary IgG excretion was higher in diabetic patients compared with healthy controls, and its excretion in diabetic patients with normoalbuminuria could predict the development of microalbuminuria(Narita et al. 2006). One study showed that significant correlations between diurnal changes in systolic blood pressure and those of urinary excretion of $\operatorname{IgG}$ were found in diabetic patients but not in controls, suggesting urinary excretion of $\operatorname{IgG}$ could be a more sensitive biomarker of glomerular capillary pressure change than albuminuria in normoalbuminuric diabetic patients(Hosoba et al. 2009).A recent study showed that urinary concentration of IgG2 was higher in normoalbuminuria group than in healthy subjects, and further elevations of IgG2, IgG4 and IgA were the most pronounced in microalbuminuria groups, while fractional excretion of IgG2 was the highest among all the immunoglobulins, which indicated that increases of those particular immunoglobulin subtypes could be novel biomarkers in early $\mathrm{DN}$, different from charge and size barrier impairment(Gohda et al. 2012).IgG4, one IgG isoform, has been used more specifically as a marker of glomerular charge impairment.Urinary $\mathrm{IgG} 4$ excretion was upregulated in patients with microalbuminuria, while the excretion of $\mathrm{IgG}$ and $\mathrm{IgG} 4$ were increased in patients with overt proteinuria compared with normoalbuminuric patients(Bangstad et al. 1992).

\section{TYPE IV COLlageN}

Type IV collagen is the major component released from the glomerular and tubular basement membranes as well as mesangial matrix. Urinary type IV collagen was upregulated in normoalbuminuric and microalbuminuric type 2 diabetic patients compared with healthy controls, and increased urinary type IV collagen was significantly correlated with the amount of albuminuria but not with $\mathrm{HbA}_{1} \mathrm{C}$ (Kado et al. 1996, Sthaneshwar and Chan 2010). An early study showed that urinary type IV collagen was more sensitive than urinary albumin as a marker for early DN(Kotajima et al. 2000), another study showed that urinary type IV collagen excretion increased with microalbuminuria and negatively correlated with creatinine clearance(Tan et al. 2002). Another conflicting follow-up study showed that urinary type IV collagen levels were higher in patients with microalbuminuria than in normoalbuminuric patients and correlated with eGFR and urinary albumin excretion rate, but urinary type IV collagen level at baseline was not associated with progression to a higher stage of DN and only suggested renal function deterioration in type 2 diabetic patients without overt proteinuria(Araki et al. 2010). 


\section{Podocytes And Podocyte-Associated Molecules}

Podocytes are key structural components of the glomerular filtration barrier. It is widely accepted that glomerular podocyte number declines plus urinary excretion of podocytes play an important role in the progression of DN (Weil et al. 2011). Urinary podocytes can be detected in diabetic patients with microalbuminuria and overt albuminuria (Nakamura et al. 2000), monitoring urinary podocytes can reveal potential urinary markers for early diagnosis of DN(Dalla Vestra et al. 2003, Wang et al. 2013).Studies showed that podocyte specific protein synaptopodin, podocin and nephrin were downregulated in DN renal biopsies, urinary nephrin levels (nephrinuria) were found to be present in the microalbuminuric, macroalbuminuric patients and normoalbuminuric patients at different ratios, suggesting that nephrinuria might be an early biomarker of DN(Patari et al. 2003, Jim et al. 2012). Urinary podocalyxin were found to be elevated in $53.8 \%$ patients at normoalbuminuric stage, $64.7 \%$ at the microalbuminuric stage and $66.7 \%$ at overt proteinuric stage, which suggested that urinary podocalyxin could be a biomarker for detecting early podocyte injury of DN(Hara et al. 2012). A recent report showed that urinary exosomal Wilms tumor-1 protein (WT-1) level was higher in patients with proteinuria associated with increased urine protein-to-creatinine ratio, serum creatinine and eGFR decline, which suggested that urinary WT-1 upregulation could be useful as early non-invasive marker for DN(Kalani et al. 2013). Another study of urinary podocyte-associated mRNA profile in different stages of DN showed that mRNA expression of podocalyxin, CD2-AP, $\alpha$-actin 4 and podocin were upregulated with the progression of DN, suggesting that quantification of urinary podocyte-associated molecules could be useful biomarkers of DN(Zheng et al. 2011). In experimental diabetic rats elevated concentrations of Amadori-modified glycated albumin (AGA) were linked with increased nephrin, podocalyxin and $\beta$ ig-h3 protein, test compound treatment can reduce the progression of DN, indicating a new therapeutic target of DN(Cohen and Shearman 2013). Vascular endothelial growth factor A (VEGF-A) has been implicated to be upregulated in DN as another podocyte-derived biomarker, which was paralleled by an increase in urinary VEGF protein level and soluble form of VEGFR-1 (sFkt-1) in type 2 diabetic microalbuminuric patients, in this study urinary excretion of VEGF was significantly higher even at the normoalbuminuric stage and increased with the progression of DN (Kim et al. 2005).

\section{TRANSFERRIN}

Transferrin is a plasma protein similar to the albumin weight (MW $76.5 \mathrm{kDa}$ ). It is expected to be more filtered through glomerular barrier and excreted in urine for being less anionic than albumin. Although urinary transferrin excretion is not specific for DN because its increase could be found in primary glomerulonephritis(Mackinnon et al. 2003), several reports have suggested that urinary transferrin excretion could be a more sensitive marker of DN than the gold standard microalbuminuria(Hong and Chia 1998, Narita et al. 2006), and a systemic review including 13 valid studies indicated that urinary transferrin excretion was a good marker for predicting the onset of DN (Hellemons et al. 2012). Some studies have reported that urinary transferrin excretion was upregulated in type 2 diabetic patients compared with healthy controls, even in normoalbuminuric cases, others suggested increased urinary transferrin excretion could predict the development of microalbuminuria in type 2 diabetic patients with normoalbuminuria(Zhou et al. 1997, Narita et al. 2004).

\section{Ceruloplasmin}

Ceruloplasmin (MW $151 \mathrm{kDa}$ ) is the main plasma copper-carrying protein and more negatively charged than albumin making it difficult to be filtered by glomerular capillary. Urinary ceruloplasmin was detected in normoalbuminuric diabetic patients, upregulated ceruloplasmin in urine played a predictive role for the development of microalbuminuria in normoalbuminuric diabetic patients(Narita et al. 2004, Narita et al. 2006, Ohara et al. 2014). The urinary ratio of ceruloplasmin and creatinine was higher in type $2 \mathrm{DN}$ subgroup compared with non-DN subgroup, of which the ratio had $91.4 \%$ of sensitivity, $61.4 \%$ of specificity in the early diagnosis of DN(Qin et al. 2004). An early study showed that urinary excretion of ceruloplasmin was significantly increased in overt proteinuria patients, 


\section{Li-Hualiu et al.}

urinary $\alpha 1$-microglobulin and $\mathrm{N}$-acetyl- $\beta$-D-glucosaminidase (NAG) were increased also in these patients, suggesting urinary ceruloplasmin could be used as a biomarker of renal tubulointerstitial injury in diabetic patients(Ito et al. 2008). A newly published systemic review showed that urinary ceruloplasmin was considered to be valid marker predicting the onset of DN (Hellemons et al. 2012). The data mentioned above indicated that urinary ceruloplasmin could be a promising biomarker of DN but mainly limited in type 2 diabetes, further investigations should be conducted to confirm the diagnostic value compared to albuminuria in type 1 diabetes.

\section{GroWth FACTORS}

\subsection{Transforming Growth Factor-Beta (TGF-B)}

Transforming growth factor-beta (TGF- $\beta$ ) is a key inducer of extracellular matrix proteins and is generally recognized as an important growth factor in the pathogenesis of DN. TGF- $\beta$ can induce an extracellular matrix protein h3 ( $\beta$ ig-h3) and the ratio of $\beta$ ig-h3 to creatinine in urine was found to be elevated in type 2 diabetic patients with normo-, micro- and macro-albuminuria compared with the healthy controls(Ha et al. 2004). Urinary levels of TGF- $\beta$ have been found to increase in both young type 1(Korpinen et al. 2000) and type 2(Sharma et al. 1997) diabetic patients, and DN patients had higher urinary TGF- $\beta$ levels than normoalbuminuric diabetic patients and healthy controls(Gilbert et al. 2001). All these finding mentioned above indicate that urinary TGF- $\beta$ and TGF- $\beta$ induced protein could be biomarkers of the progression of DN.

\subsection{Connective Tissue Growth Factor (CTGF)}

Connective tissue growth factor (CTGF) has been recognized as a profibrotic cytokine acting as downstream of TGF- $\beta$ and shown to promote renal fibrosis. One cross-sectional study showed that urinary CTGF levels were increased and correlated with urinary albumin excretion in type $1 \mathrm{DN}$ patients (Nguyen et al. 2006). A subsequent prospective study in both type 1 and type 2 diabetic patients after 6 years follow-up, urinary CTGF levels were upregulated and correlated with the progression of microalbuminuria, suggesting urinary CTGF level could be a prognostic biomarker for early progression of DN(Tam et al. 2009).

\section{Filtered Plasma PROTEINS: A1MG, RBP, B2MG}

Urinary excretion of low molecular weight plasma proteins include $\alpha_{1}$-microglobulin $\left(\alpha_{1} \mathrm{MG}, \mathrm{MW}\right.$ up to $31 \mathrm{kDa}$ ), retinol-binding protein (RBP, MW $21.4 \mathrm{kDa})$ and $\beta_{2^{-}}$microglobulin $\left(\beta_{2} \mathrm{MG}\right.$, MW 11.8 $\mathrm{kDa}$ ), which were filtered through the glomerulus and were nearly entirely reabsorbed by proximal renal tubule(Hong and Chia 1998). Urinary RBP(Lapsley et al. 1993) and $\alpha_{1} \mathrm{MG}$ were elevated in diabetic patients with normal UAE(Hong et al. 2003). Urinary RBP excretion increased concomitantly with that of NAG in type 2 diabetic patients(Shimizu et al. 1992), and urinary $\alpha_{1}$ MG levels have been associated or correlated with both transferrin(O'Donnell et al. 1991) and albumin(Hong et al. 2003) excretion (two markers of glomerular damage). Another interesting study showed that urinary $\alpha_{1} \mathrm{MG}$ excretion was not increased significantly between normoalbuminuric diabetic patients and healthy controls(Kalansooriya et al. 2007). These findings suggested that urinary RBP and $\alpha_{1} M G$ measuring proximal tubular dysfunction could be useful markers for the early detection of DN(Hong et al. 2003) complementing markers of glomerular dysfunction such as albumin rather than outperforming it(Matheson et al. 2010). Conversely the use of $\beta_{2} \mathrm{MG}$ for the detection of DN may be more uncertain. Urinary $\beta_{2} \mathrm{MG}$ excretion has been reported to be associated with $\mathrm{DN}$ (Hong et al. 2000), however conflicting results have been reported with both the difference in urinary $\beta_{2} \mathrm{MG}$ levels between diabetic patients and controls(Tanaka et al. 1989, Groop et al. 1990), variation of urine PH may explain the divergence in these experimental results because $\beta_{2} \mathrm{MG}$ can degrade below PH 5.5 at the room temperature or below PH 6 at body temperature. Because of instability of $\beta_{2} \mathrm{MG}$, some studies recommended the use of RPB and $\alpha_{1}$ MG as sensitive indicators of tubular damage(Flynn 1990, Matheson et al. 2010). 


\section{Ngal, KIM-1 AND NAg}

Neutrophil gelatinase-associated lipocalin (NGAL), a $25 \mathrm{kDa}$ protein of lipocalin family from human neutrophils, was covalently associated with gelatinase, which was produced in the distal nephron and increased in response to kidney injury. Kidney injury molecule 1 (KIM-1), a transmembrane protein on the apical membrane of PTCs, was increased also in acute kidney injury. $\mathrm{N}$-acetyl- $\beta$-D-glucosaminidase (NAG), an enzyme (MW 130kDa) involved in carbohydrate metabolism, plasma NAG can't be filtered through kidney and presence in urine is exclusively secreted by tubular cells lysosomes(Hong and Chia 1998). Urinary NGAL, KIM-1 and NAG in type 2 diabetic patients were markedly increased(Fu et al. 2012). Urinary NGAL and KIM-1 were found to be upregulated in both type 1 diabetic patients with or without albuminuria and in type 2 diabetic patients in accordance with the decline in eGFR(Nielsen et al. 2010, Nielsen et al. 2012). An early study showed that urinary NAG and NGAL were significantly elevated in normoalbuminuric diabetic patients compared with nondiabetic healthy control and were associated with albuminuria and eGFR independent of age, sex and plasma concentrations of the respective biomarkers(Nauta et al. 2011). Another recent cross-sectional study for the purpose of investigating the prevalence of tubular damage of type 2 diabetes showed that urinary NGAL was strongly positively correlated with the urinary albumin/creatinine ratio and negatively correlated with eGFR, where urinary KIM-1 showed no significant difference among the three diabetic subgroups (normoalbuminuria, microalbuminuria and overt proteinuria groups) but not correlated with albumin/creatinine ratio or eGFR(Fu et al. 2012). An early study showed a stepwise increase in NAG concentration from normo- to micro- and macro-albuminuric patients(Piwowar et al. 2006). All the data mentioned above indicated that tubular damage is common at the early stage of type 2 diabetic patients and urinary NGAL, KIM-1 and NAG could be early promising biomarkers for renal impairment in DN patients. A recent study showed that urinary KIM-1, NAG and NGAL were not only urinary biomarkers of renal tubular injury, but also showed that median concentrations of $\mathrm{KIM}-1 / \mathrm{Cr}, \mathrm{NAG} / \mathrm{Cr}$ and $\mathrm{NGAL} / \mathrm{Cr}$ were highest in diabetic patients with macroalbuminuria and $\mathrm{NGAL} / \mathrm{Cr}$ was positively associated with ESRD and mortality(Fufaa et al. 2014).

\section{OXIDATIVE STRESS Biomarker}

Oxidative stress is widely recognized to be a key factor contributing to the development and progression of DN. Polyol pathway activation, advanced glycation end product overproduction and the protein kinase $\mathrm{C}$ isoforms activation were found to participate in the pathogenesis of DN. An early report showed that urinary pentosidine concentration was greatly increased in type 2 diabetic patients with overt nephropathy and correlated with urinary $\beta_{2} \mathrm{MG}$ excretion, which suggested petosidine could be a urinary marker of $\mathrm{DN}$ (Aso et al. 2004). 8-Hydroxy-2'-deoxyguanosine (8-OHdG) and 8-oxo-7,8-dihydro-2'-deoxyguanosine (8-oxodG) are products of the oxidative DNA damage could be freely filtered, and urinary levels could act as index of systemic oxidative stress(Lee and Choi 2014). Urinary excretion of $8-\mathrm{OHdG}$ was markedly increased in type 2 diabetic patients compared to the healthy control, a high level of urinary 8-OHdG was found to be associated with high glycosylated hemoglobin(Leinonen et al. 1997) as well as the severity of tubulointerstitial damage(Kanauchi et al. 2002). Recent studies showed that the patients with higher urinary excretion of 8-oxodG compared to the patients with moderate or lower excretion of 8-oxodG suggested that urinary 8-oxodG could be a useful clinical biomarker to predict the development of DN(Hinokio et al. 2002, Broedbaek et al. 2011). Taken together, these findings suggested that urinary 8-OHdG and 8-oxodG could be useful biomarkers to predict the development and progression of DN despite a recent conflicting reported result(Serdar et al. 2012).

\section{Liver-Type FatTy ACID Binding Protein (L-FABP)}

Liver-type fatty acid binding protein (L-FABP), a $14 \mathrm{kDa}$ small molucule, is expressed in renal proximal tubular cells and assumed to be shed into urine in response to hypoxia by decreased peritubular capillary blood flow(Kamijo et al. 2006) and can be recognized as excellent biomarkers in the urine for the early prediction of acute kidney injury(McMahon and Murray 2010). Two studies on 
type 1 diabetes and five studies on type 2 diabetes have reported the relationship between urinary L-FABP concentrations and the severity of DN(Kamijo-Ikemori et al. 2013, Fufaa et al. 2014, Kim et al. 2014). In type 1 diabetes, urinary L-FABP concentrations were upregulated with the progression of DN and were higher in normoalbuminuric patients than in healthy controls(Nielsen et al. 2009, Panduru et al. 2013), these findings suggested that urinary L-FABP reflected DN severity accurately and could be a suitable biomarker for early detection of DN(Kamijo-Ikemori et al. 2013). In type 2 diabetes, several studies showed increases of urinary L-FABP concentrations with the progression of DN(Nakamura et al. 2005, Suzuki et al. 2005, Kamijo-Ikemori et al. 2011, Kim et al. 2014) but one study showed confilicting result(Fufaa et al. 2014). One study reported that urinary L-FABP concentrations were higher in patients with normoalbuminuria than in healthy controls(Kamijo-Ikemori et al. 2011) and there are different results between the various degrees of albminuria ranging from normo- to micro subgroups, increased urinary L-FABP was not correlated with a decline in GFR. All these findings suggested that urinary L-FABP could be a predictor of the progression of DN or a target of therapeutic regimens but needs further multicenter clinical studies on large populations to confirm the clinical usefulness(Kamijo-Ikemori et al. 2013). All the studies were performed in patients without albuminuria, which suggested L-FABP could be useful in early stages of DN but not late stages(Kamijo-Ikemori et al. 2011).

\section{INFLAMMATION BIOMARKERS: TNF-A, MCP-1, IL-6}

Growing evidences showed that pro-inflammatory cytokines have been implicated in the pathogenesis of DN, therefore measurement of pro-inflammatory cytokines in urine might help in the diagnosis of DN.

\subsection{Tumor Necrosis Factor Alpha (TNF-A)}

Tumor necrosis factor alpha (TNF- $\alpha$ ) is a key inflammation mediator in the pathogenesis of kidney injury by promoting apoptosis, inflammation and extracellular matrix accumulation, by increasing albumin permeability and reducing GFR. The major source of TNF- $\alpha$ are activated macrophages, intrinsic renal cells can also be able to express TNF- $\alpha$ contributing to renal injury(Timoshanko et al. 2003). An early study showed that urinary TNF- $\alpha$ excretion was increased in type 2 diabetic patients with micro- and macro-albuminuria compared with healthy controls, also increased with the severity of nephropathy gradually(Navarro et al. 2008). Urinary TNF- $\alpha$ excretion was associated with urinary albumin and NAG excretion and correlated with the severity of DN in terms of both glomerular and tubular damages(Navarro et al. 2003, Navarro et al. 2006). Taken together, these findings indicated that TNF- $\alpha$ could be a potential urinary biomarker of DN.

\subsection{Monocyte Chemoattractant Protein-1 (MCP-1)}

Urinary MCP-1 excretion increased in accordance with the DN level and could be useful in evaluating the degree of renal injuries in type 2 DN patients(Tashiro et al. 2002), and one report showed that urinary MCP-1/creatinine in overt proteinuria was higher than patients with normoalbuminuria and microalbuminuria, and urinary MCP-1 was correlated with the decline of eGFR(Tam et al. 2009). A recent study showed that urinary MCP-1 was significantly higher in patients with doubling of serum creatinine or death, in the Cox regression, urinary MCP-1 excretion was positively associated with the risk of serum creatinine doubling or death, and urinary MCP-1 remained as significant independent predictor of this primary outcome (doubling of serum creatinine or death)(Titan et al. 2012). All the data mentioned above indicated that urinary $\mathrm{MCP}-1$ could be a prognostic biomarker for progression of DN, further investigations need to be performed to confirm whether urinary MCP-1 has a role in the setting of normoalbuminuria and microalbuminuria in DN(Wang et al. 2013).

\subsection{Interleukin 6 (Il-6)}

In an earlier report urinary IL-6 concentration was increased significantly and associated with the progression of DN in type 2 diabetic patients(Shikano et al. 2000). But one study showed that urinary IL-6 level was not increased significantly between the different stages of albuminuria, only with a 
slight increase between patients with micoralbuminuria and normoalbuminuria(Navarro et al. 2008). Although a recent systemic review announced that IL- 6 could be one of the 13 valid biomarkers for predicting progression of $\mathrm{DN}($ Hellemons et al. 2012), but there are conflicting results regarding the application of IL-6 in the early diagnosis of DN(Moresco et al. 2013), more studies seem necessary to confirm the use of measuring urinary IL-6 levels in the diagnosis or monitoring of DN.

\subsection{Vitamin D-Binding Protein (VDBP)}

Vitamin D-binding protein (VDBP), known as gc-globulin, can bond and transport vitamin D throughout the body. Clinically exaggerated excretion of urinary VDBP was associated with tubular dysfunction(Thrailkill et al. 2011). A recent study showed that urinary VDBP excretion levels were elevated significantly in patients of DN 1 (microalbuminuria) and DN 2 (macroalbuminuria) groups compared with those of the DM group and healthy controls(Tian et al. 2014). These findings suggested urinary VDBP could be a potential biomarker for early detection and prevention of DN.

\section{SERUM BIOMARKERS FOR DN}

\subsection{Low molecular weight proteins (LMWPs)}

Serum creatinine (for estimation of GFR) as well as urinary albumin excretion (UAE) are recommended in all adults with diabetes by the American Diabetes Association and the National Kidney Foundation(2007, Kdoqi 2007). GFR is generally estimated by the Cockroft-Gault formula or the abbreviated MDRD equation, and the MDRD formula needs to be applied cautiously because serum creatinine levels are affected by diverse factors including age, gender, race and body composition, thus there are limits in GFR estimation from serum creatinine. Low molecular weight proteins (LMWPs) have been proposed recently as an alternative for the GFR estimation. Serum LMWPs levels could reflect changes in the glomerular filtration and several studies have shown measurement of the serum concentration of LMWPs could be used for GFR estimation, especially serum cystatin-C, $\beta_{2} \mathrm{MG}$, Angptl2 and sKlotho have been proven to be more accurate indicators for GFR estimation compared with serum creatinine(Ito et al. 2008).

\subsection{Serum Cystatin-C}

Cystatin- $\mathrm{C}$ is produced by the nucleated cells and exhibits stable production rate even in inflammatory conditions(Abrahamson et al. 1990). Cystatin-C is not influenced by age, gender, or muscle mass, serum cystatin-C concentration has been regarded as a desirable endogenous parameter for GFR. Automated immunoassays have been developed based on PETIA or PENIA. A meta-analysis has shown that GFR was strongly correlated with cystatin-C measured by PENIA(Dharnidharka et al. 2002). Several studies about comparing cystatin-C with serum creatinine showed that correlation between cystatin-C and GFR should be stronger than that between serum creatinine and GFR(Dharnidharka et al. 2002, Laterza et al. 2002). Another study showed that serum cystatin-C change is strongly correlated with iotharamate clearance in measuring GFR in diabetic patients, which is better than GFR measured by serum creatinine(Perkins et al. 2005). A recent study showed that serum cystatin-C is better correlated with GFR than serum creatinine, the Cockroft-Gault formula and the MDRD equation in diabetic patients (Pucci et al. 2007). A newly published report showed that serum cystatin-C based equation was less biased and could predict GFR changes better than the MDRD equation(Beauvieux et al. 2007). Another study showed that cystatin-C is the most sensitive and specific marker in DN for the follow-up and monitoring renal damage progression(Assal et al. 2013). Taken together, these findings indicated that serum cystatin-C could be a replacement to serum creatinine for measuring GFR in diabetic patients.

\subsection{Serum $\beta_{2}$ MG}

An early study showed that serum $\beta_{2} \mathrm{MG}$ concentration was increased and correlated with the GFR decline, which was better than that of serum creatinine(Bianchi et al. 2001). And in type 2 diabetic patients with moderately decreased GFR, another study showed that serum $\beta_{2}$ MG concentration may serve as a more sensitive biomarker for the changes of GFR than serum creatinine(Mojiminiyi and 
Abdella 2003). A recent study had similar results that serum $\beta_{2} \mathrm{MG}$ concentration was significantly higher in normoalbuminuric and microabluminuric type 2 diabetic patients compared with controls and serum $\beta_{2}$ MG was significantly inversely correlated with GFR values(Aksun et al. 2004). And there was an converse study result showed that serum $\beta_{2}$ MG diagnostic value was similar to that of serum creatinine(Donadio et al. 2001). A recent study confirmed serum $\beta_{2} \mathrm{MG}$ level was a novel predictor of diabetes related mortality in diabetic patients regardless of renal function (Cheung et al. 2013). These findings suggest that serum $\beta_{2} \mathrm{MG}$ could be applied as an indicator of GFR in diabetic patients despite serum $\beta_{2} \mathrm{MG}$ levels could be altered by multiple factors including age, gender, liver disease, immune disorders and malignant disease(Viberti et al. 1981).

\subsection{Serum Angiopoietin-Like Protein 2 (Angptl2)}

Angiopoietin-like protein 2 (Angpt12), a member of the ANGPTL family, is a stress responsive adipose tissues-secreted protein, has been reported to express abundantly in heart and adipose tissues and a key mediator linking obesity to adipose tissue inflammation, systemic insulin resistance and metabolic syndrome in human and mice(Oike and Tabata 2009, Tabata et al. 2009, Ouchi et al. 2011). In the experimental study showed that replenishment of Angptl2 could stimulate insulin sensitivity and improve the type 2 diabetic state (Kitazawa et al. 2011). An early study showed that Angptl2 was expressed more frequently in diabetic glomeruli compared to donor controls, indicating Angptl2 could play an important role in the pathogenesis of $\mathrm{DN}$ (Sun et al. 2007). A recent study showed that serum Angptl2 were elevated in diabetic patients with normoalbuminuria, microalbuminuria and macroalbuminuria than that of healthy controls, in which Angpt12 levels were correlated with urinary ACR(Li et al. 2013). Taken together, serum Angptl2 could be a biomarker of the progression of DN.

\subsection{Serum Soluble-Klotho (Sklotho)}

Serum soluble Klotho (sKlotho), an anti-aging phosphaturic protein, is associated with vascular-renal protection. Two recent study showed that serum sKlotho levels were significantly decreased in diabetic patients with normo-, micro- and macroalbuminuria compared with controls and negatively correlated with UACR(Lee et al. 2014, Wu et al. 2014), and urinary soluble $\alpha$-Klotho levels were increased also(Lee et al. 2014), while an early study showed that serum sKlotho in diabetic patients was decreased in the presence of microalbuminuria and correlated with VEGF-A(Kacso et al. 2012), these data suggested serum sKlotho may become new biomarker of the early diagnosis of type $2 \mathrm{DN}$.

\section{EPIGENETICS FOR DN BIOMARKER}

The epigenetics has been widely used in the study for the DN mechanism research. Epigenetics includes the heritable changes occurring outside the modification of DNA coding sequence such as programmed changes during embryonic development(Dressler 2008) and structural adaptation of chromosomal regions in response to the appropriate signals(Bonasio et al. 2010). Epigenetics is also responsible for the elementary ability for the cell to "memorize" the past events, which is relevant in diabetes and its complications, and experimental and clinical studies demonstrate the happening of the "metabolic memory" caused by the prior exposure to hyperglycemia, which could result in persistent risk for developing complications despite intensive glucose control(Pirola et al. 2010, Villeneuve and Natarajan 2010). These findings force us to focus epigenetic mechanisms on the studies of biomarkers in the DN pathogenesis.

Epigenetics include DNA methylation, post-translational modifications of histone amino-terminal tails and noncoding RNAs. Posttranslational modifications can occur via methylation, acetylation and phosphorylation which can regulate gene expression. DNA methylation has been fully studied in DN so far including regulating insulin expression because mouse embryonic stem cells were shown to be demethylated when they differentiate into insulin-expressing cells(Kuroda et al. 2009). Epigenetics could also explain a model of intrauterine growth retardation leading to type 2 diabetes via silencing Pdx1, an important transcription factor of insulin gene expression(Park et al. 2008).

Another epigenetics branch is short, noncoding RNAs (microRNAs, miRNAs) that can regulate gene 
expression. miRNAs have divers roles in lots of cellular and physiological activities such as growth, cell cycle, proliferation, metabolism and apoptosis. miRNAs are important in maintaining renal homeostasis and kidney disease. In vitro and in vivo studies of miRNAs have shown critical roles in the development of DN and in the progression of renal fibrosis(Schena et al. 2014). MiRNAs are present in multiple body fluids and are highly stable and resistant to storage handling, which make them attractive to use as biomarkers(Patnaik et al. 2010, Weber et al. 2010). Lots of work has been dedicated into trying to understand how miRNAs regulating and regulated by factors contributing to DN(Kantharidis et al. 2011, McClelland et al. 2014, McClelland and Kantharidis 2014). Recent reviews have analyzed the effects of miRNAs on DN from normal kidney function(Chandrasekaran et al. 2012, Khella et al. 2013), to glomerular podocyte dysfunction(Kato et al. 2012), kidney fibrosis(Srivastava et al. 2013), the RAS system, AGE/RAGE signaling and under oxidative stress(Hagiwara et al. 2013) as well as kidney inflammation(McClelland and Kantharidis 2014). Much work has been performed on the role of miR-192 in cell culture and human studies of DN although with contradictory results. MiR-192 was induced by TGF- $\beta$ in cultured mesangial cells and diabetic kidneys through the repressor protein SIP1 leading to increased collagen deposition in the mesangium(Kato et al. 2007). However two seminal studies in humans have found reduced miR-192 expression in renal biopsy samples and correlated with GFR decrease and the degree of loss with tubulointerstitial fibrosis(Krupa et al. 2010). Interesting results showed that TGF- $\beta$ treating proximal tubular cells can decrease miR-192 expression suggesting decreased miR-192 was mediated by TGF- $\beta$. It was confirmed by another study showing a decreased miR-192 and miR-215 expression in the mesangial and proximal tubular cells after TGF- $\beta$ treatment. More recently, TGF- $\beta$ signaling pathway has been implicated important in renal fibrosis, with upregulation of miR-21 and miR-192, while downregulation of miR-29 and miR-200(Lan 2012). In addition, miR-29 could regulate both HDAC actions and nephrin acetylation and contribute to podocyte homeostasis as well as renal function(Lin et al. 2014). The study showed that miR-29a overexpression could attenuate nephrin ubiquitination, HDAC4 signaling, and urinary nephrin excretion associated with diabetes and restored nephrin acetylation, demonstrating increased miR-29a may protect against diabetic podocytopathy(Lin et al. 2014). A recent study showed that miR-451 could play protective role in early DN by suppressing mesangial hypertrophy and downregulating p38 MAPK signaling (Zhang et al. 2012). While miR-195 decrease in early DN to protect mesangial cells from apoptosis and induce mesangial proliferation, whereas miR-195 increase in advanced stages of DN by favoring podocyte apoptosis and proteinuria(DiStefano et al. 2013). A new report showed a significant deregulation of miR-21, miR-210 and miR-126 in urine and plasma in type 1 diabetic pediatric patients indicating an early onset of DN(Osipova et al. 2014). Moreover, miRNAs use as biomarkers of DN for diagnosis and follow-up (Alvarez and Distefano 2013, Kato et al. 2013) as well as potential therapeutic targets(DiStefano et al. 2013) have been further reviewed elsewhere. In the future, identification of additional miRNAs may further enhance these efforts focused on harnessing the diagnostic and interventional potential of miRNAs for DN.

\section{Conclusions ANd Perspectives}

During the past few decades, there has been vast interest in discovering DN biomarkers allowing for the detection of DN early stages and progressive renal function decline in diabetic patients, but we still lack good DN predictive biomarkers. Recent tremendous studies have highlighted lots of promising new biomarkers in urine, serum and kidney tissues for early DN diagnosis and prediction of DN progression, which have identified lots of new and important biomarkers but not yet applied to replace the use of microalbuminuria in clinical practice, which still is the gold standard for detecting DN.

A number of clinical and experimental studies exploring serum or urinary biomarkers for detecting DN biomarkers are discussed in this review. Urinary biomarkers could be sensitive, noninvasive and clinically useful method which is particularly attractive because urine is the easily available source. Newly reported urinary ACE2 levels were increased and correlated with albuminuria, serum 


\section{Li-Hualiu et al.}

creatinine and glucagon, suggesting that urinary ACE2 could be used as a biomarker for DN(Salem et al. 2014), and vast similar results have been reported. But because of these kinds of studies have limitations such as small sample size, cross-sectional, short-term prospective nature and the long way from bench to bed, new molecular technologies such as transcriptomics, genetics and genomics should be further used to discover novel DN biomarkers despite the increased costs. Unfortunately, despite the tremendous number of biomarkers have been discovered, none of them has been proven to be superior to albuminuria in this review. Further studies should be performed in both searching and validating new biomarkers and conducting larger longitudinal prospective studies to verity the applications of these biomarkers as more predictive, reliable, accurate, inexpensive and prognostic markers for early diagnosis and progression of DN.

\section{Conflict of Interests}

The authors have no conflict of interests to declare.

\section{ACKNOWLEDGEMENT}

This study was supported in part by Natural Science Foundation Project of Jilin provincial Science \& Technology (201215067), International Cooperation Project of Jilin provincial Science \& Technology (20140414030GH), Health Department Project of Jilin province (2011Z025) and Basic Scientific Research Business expenses Project of Jilin University (201103053).

\section{REFERENCES}

[1]. Abrahamson, M., I. Olafsson, A. Palsdottir, M. Ulvsback, A. Lundwall, O. Jensson and A. Grubb (1990). "Structure and expression of the human cystatin C gene." Biochem J 268(2): 287-294.

[2]. Aksun, S. A., D. Ozmen, B. Ozmen, Z. Parildar, I. Mutaf, N. Turgan, S. Habif, K. Kumanliogluc and O. Bayindir (2004). "Beta2-microglobulin and cystatin C in type 2 diabetes: assessment of diabetic nephropathy." Exp Clin Endocrinol Diabetes 112(4): 195-200.

[3]. Alvarez, M. L. and J. K. Distefano (2013). "The role of non-coding RNAs in diabetic nephropathy: potential applications as biomarkers for disease development and progression." Diabetes Res Clin Pract 99(1): 1-11.

[4]. Araki, S., M. Haneda, D. Koya, K. Isshiki, S. Kume, T. Sugimoto, H. Kawai, Y. Nishio, A. Kashiwagi, T. Uzu and H. Maegawa (2010). "Association between urinary type IV collagen level and deterioration of renal function in type 2 diabetic patients without overt proteinuria." Diabetes Care 33(8): 1805-1810.

[5]. Aso, Y., K. Takanashi, K. Sekine, N. Yoshida, K. Takebayashi, K. Yoshihara and T. Inukai (2004). "Dissociation between urinary pyrraline and pentosidine concentrations in diabetic patients with advanced nephropathy." J Lab Clin Med 144(2): 92-99.

[6]. Assal, H. S., S. Tawfeek, E. A. Rasheed, D. El-Lebedy and E. H. Thabet (2013). "Serum cystatin $\mathrm{C}$ and tubular urinary enzymes as biomarkers of renal dysfunction in type 2 diabetes mellitus." Clin Med Insights Endocrinol Diabetes 6: 7-13.

[7]. Bakoush, O., J. Tencer, J. Tapia, B. Rippe and O. Torffvit (2002). "Higher urinary IgM excretion in type 2 diabetic nephropathy compared to type 1 diabetic nephropathy." Kidney Int 61(1): 203-208.

[8]. Bangstad, H. J., A. Kofoed-Enevoldsen, K. Dahl-Jorgensen and K. F. Hanssen (1992). "Glomerular charge selectivity and the influence of improved blood glucose control in type 1 (insulin-dependent) diabetic patients with microalbuminuria." Diabetologia 35(12): 1165-1169.

[9]. Beauvieux, M. C., F. Le Moigne, C. Lasseur, C. Raffaitin, C. Perlemoine, N. Barthe, P. Chauveau, C. Combe, H. Gin and V. Rigalleau (2007). "New predictive equations improve monitoring of kidney function in patients with diabetes." Diabetes Care 30(8): 1988-1994.

[10]. Bianchi, C., C. Donadio, G. Tramonti, C. Consani, P. Lorusso and G. Rossi (2001). "Reappraisal of serum beta2-microglobulin as marker of GFR." Ren Fail 23(3-4): 419-429.

[11]. Bonasio, R., S. Tu and D. Reinberg (2010). "Molecular signals of epigenetic states." Science 330(6004): 612-616. 
[12]. Broedbaek, K., A. Weimann, E. S. Stovgaard and H. E. Poulsen (2011). "Urinary 8-oxo-7,8-dihydro-2'-deoxyguanosine as a biomarker in type 2 diabetes." Free Radic Biol Med 51(8): 1473-1479.

[13]. Chandrasekaran, K., D. S. Karolina, S. Sepramaniam, A. Armugam, E. M. Wintour, J. F. Bertram and K. Jeyaseelan (2012). "Role of microRNAs in kidney homeostasis and disease." Kidney Int 81(7): 617-627.

[14]. Cheung, C. L., K. S. Lam and B. M. Cheung (2013). "Serum beta-2 microglobulin predicts mortality in people with diabetes." Eur J Endocrinol 169(1): 1-7.

[15].Cohen, M. P. and C. W. Shearman (2013). "Inhibiting Amadori-modified albumin formation improves biomarkers of podocyte damage in diabetic rats." Physiol Rep 1(4): e00083.

[16].Dalla Vestra, M., A. Masiero, A. M. Roiter, A. Saller, G. Crepaldi and P. Fioretto (2003). "Is podocyte injury relevant in diabetic nephropathy? Studies in patients with type 2 diabetes." Diabetes 52(4): 1031-1035.

[17]. Dharnidharka, V. R., C. Kwon and G. Stevens (2002). "Serum cystatin C is superior to serum creatinine as a marker of kidney function: a meta-analysis." Am J Kidney Dis 40(2): 221-226.

[18]. DiStefano, J. K., M. Taila and M. L. Alvarez (2013). "Emerging roles for miRNAs in the development, diagnosis, and treatment of diabetic nephropathy." Curr Diab Rep 13(4): 582-591.

[19]. Donadio, C., A. Lucchesi, M. Ardini and R. Giordani (2001). "Cystatin C, beta 2-microglobulin, and retinol-binding protein as indicators of glomerular filtration rate: comparison with plasma creatinine." J Pharm Biomed Anal 24(5-6): 835-842.

[20]. Dressler, G. R. (2008). "Epigenetics, development, and the kidney." J Am Soc Nephrol 19(11): 2060-2067.

[21]. Flynn, F. V. (1990). "Assessment of renal function: selected developments." Clin Biochem 23(1): 49-54.

[22]. Fu, W. J., S. L. Xiong, Y. G. Fang, S. Wen, M. L. Chen, R. T. Deng, L. Zheng, S. B. Wang, L. F. Pen and Q. Wang (2012). "Urinary tubular biomarkers in short-term type 2 diabetes mellitus patients: a cross-sectional study." Endocrine 41(1): 82-88.

[23]. Fufaa, G. D., E. J. Weil, R. G. Nelson, R. L. Hanson, J. V. Bonventre, V. Sabbisetti, S. S. Waikar, T. E. Mifflin, X. Zhang, D. Xie, C. Y. Hsu, H. I. Feldman, J. Coresh, R. S. Vasan, P. L. Kimmel and K. D. Liu (2014). "Association of urinary KIM-1, L-FABP, NAG and NGAL with incident end-stage renal disease and mortality in American Indians with type 2 diabetes mellitus." Diabetologia.

[24]. Gilbert, R. E., A. Akdeniz, T. J. Allen and G. Jerums (2001). "Urinary transforming growth factor-beta in patients with diabetic nephropathy: implications for the pathogenesis of tubulointerstitial pathology." Nephrol Dial Transplant 16(12): 2442-2443.

[25]. Gohda, T., W. H. Walker, P. Wolkow, J. E. Lee, J. H. Warram, A. S. Krolewski and M. A. Niewczas (2012). "Elevated urinary excretion of immunoglobulins in nonproteinuric patients with type 1 diabetes." Am J Physiol Renal Physiol 303(1): F157-162.

[26]. Groop, L., A. Makipernaa, S. Stenman, R. A. DeFronzo and A. M. Teppo (1990). "Urinary excretion of kappa light chains in patients with diabetes mellitus." Kidney Int 37(4): 1120-1125.

[27].Ha, S. W., H. J. Kim, J. S. Bae, G. H. Jeong, S. C. Chung, J. G. Kim, S. H. Park, Y. L. Kim, S. Kam, I. S. Kim and B. W. Kim (2004). "Elevation of urinary betaig-h3, transforming growth factor-beta-induced protein in patients with type 2 diabetes and nephropathy." Diabetes Res Clin Pract 65(2): 167-173.

[28]. Hagiwara, S., A. McClelland and P. Kantharidis (2013). "MicroRNA in diabetic nephropathy: renin angiotensin, aGE/RAGE, and oxidative stress pathway." J Diabetes Res 2013: 173783.

[29].Hara, M., K. Yamagata, Y. Tomino, A. Saito, Y. Hirayama, S. Ogasawara, H. Kurosawa, S. Sekine and K. Yan (2012). "Urinary podocalyxin is an early marker for podocyte injury in patients with diabetes: establishment of a highly sensitive ELISA to detect urinary podocalyxin." Diabetologia 55(11): 2913-2919.

[30].Hellemons, M. E., J. Kerschbaum, S. J. Bakker, H. Neuwirt, B. Mayer, G. Mayer, D. de Zeeuw, H. J. Lambers Heerspink and M. Rudnicki (2012). "Validity of biomarkers predicting onset or 
progression of nephropathy in patients with Type 2 diabetes: a systematic review." Diabet Med 29(5): 567-577.

[31]. Hinokio, Y., S. Suzuki, M. Hirai, C. Suzuki, M. Suzuki and T. Toyota (2002). "Urinary excretion of 8-oxo-7, 8-dihydro-2'-deoxyguanosine as a predictor of the development of diabetic nephropathy." Diabetologia 45(6): 877-882.

[32]. Hong, C. Y. and K. S. Chia (1998). "Markers of diabetic nephropathy." J Diabetes Complications 12(1): 43-60.

[33]. Hong, C. Y., K. Hughes, K. S. Chia, V. Ng and S. L. Ling (2003). "Urinary alpha1-microglobulin as a marker of nephropathy in type 2 diabetic Asian subjects in Singapore." Diabetes Care 26(2): 338-342.

[34]. Hong, C. Y., K. S. Chia and S. L. Ling (2000). "Urinary protein excretion in Type 2 diabetes with complications." J Diabetes Complications 14(5): 259-265.

[35]. Hosoba, M., H. Fujita, T. Miura, T. Morii, T. Shimotomai, J. Koshimura, Y. Yamada, S. Ito and T. Narita (2009). "Diurnal changes in urinary excretion of IgG, transferrin, and ceruloplasmin depend on diurnal changes in systemic blood pressure in normotensive, normoalbuminuric type 2 diabetic patients." Horm Metab Res 41(12): 910-915.

[36]. Ito, H., H. Fujita and T. Takahashi (2008). "Diagnostic biomarkers of diabetic nephropathy." Expert Opin Med Diagn 2(2): 161-169.

[37]. Jeon, Y. K., M. R. Kim, J. E. Huh, J. Y. Mok, S. H. Song, S. S. Kim, B. H. Kim, S. H. Lee, Y. K. Kim and I. J. Kim (2011). "Cystatin C as an early biomarker of nephropathy in patients with type 2 diabetes." J Korean Med Sci 26(2): 258-263.

[38].Jim, B., J. Santos, F. Spath and J. Cijiang He (2012). "Biomarkers of diabetic nephropathy, the present

[39]. Jim, B., M. Ghanta, A. Qipo, Y. Fan, P. Y. Chuang, H. W. Cohen, M. Abadi, D. B. Thomas and J. C. He (2012). "Dysregulated nephrin in diabetic nephropathy of type 2 diabetes: a cross sectional study." PLoS One 7(5): e36041.

[40]. Kacso, I. M., C. I. Bondor and G. Kacso (2012). "Soluble serum Klotho in diabetic nephropathy: relationship to VEGF-A." Clin Biochem 45(16-17): 1415-1420.

[41]. Kado, S., A. Aoki, S. Wada, Y. Katayama, N. Kugai, N. Yoshizawa and N. Nagata (1996). "Urinary type IV collagen as a marker for early diabetic nephropathy." Diabetes Res Clin Pract 31(1-3): 103-108.

[42]. Kalani, A., A. Mohan, M. M. Godbole, E. Bhatia, A. Gupta, R. K. Sharma and S. Tiwari (2013). "Wilm's tumor-1 protein levels in urinary exosomes from diabetic patients with or without proteinuria." PLoS One 8(3): e60177.

[43]. Kalansooriya, A., I. Holbrook, P. Jennings and P. H. Whiting (2007). "Serum cystatin C, enzymuria, tubular proteinuria and early renal insult in type 2 diabetes." Br J Biomed Sci 64(3): 121-123.

[44]. Kamijo, A., T. Sugaya, A. Hikawa, M. Yamanouchi, Y. Hirata, T. Ishimitsu, A. Numabe, M. Takagi, H. Hayakawa, F. Tabei, T. Sugimoto, N. Mise, M. Omata and K. Kimura (2006). "Urinary liver-type fatty acid binding protein as a useful biomarker in chronic kidney disease." Mol Cell Biochem 284(1-2): 175-182.

[45]. Kamijo-Ikemori, A., T. Sugaya, D. Ichikawa, S. Hoshino, K. Matsui, T. Yokoyama, T. Yasuda, K. Hirata and K. Kimura (2013). "Urinary liver type fatty acid binding protein in diabetic nephropathy." Clin Chim Acta 424: 104-108.

[46]. Kamijo-Ikemori, A., T. Sugaya, T. Yasuda, T. Kawata, A. Ota, S. Tatsunami, R. Kaise, T. Ishimitsu, Y. Tanaka and K. Kimura (2011). "Clinical significance of urinary liver-type fatty acid-binding protein in diabetic nephropathy of type 2 diabetic patients." Diabetes Care 34(3): 691-696.

[47]. Kanauchi, M., H. Nishioka and T. Hashimoto (2002). "Oxidative DNA damage and tubulointerstitial injury in diabetic nephropathy." Nephron 91(2): 327-329.

[48]. Kantharidis, P., B. Wang, R. M. Carew and H. Y. Lan (2011). "Diabetes complications: the microRNA perspective." Diabetes 60(7): 1832-1837. 
[49]. Kato, M., J. T. Park and R. Natarajan (2012). "MicroRNAs and the glomerulus." Exp Cell Res 318(9): 993-1000.

[50]. Kato, M., J. Zhang, M. Wang, L. Lanting, H. Yuan, J. J. Rossi and R. Natarajan (2007). "MicroRNA-192 in diabetic kidney glomeruli and its function in TGF-beta-induced collagen expression via inhibition of E-box repressors." Proc Natl Acad Sci U S A 104(9): 3432-3437.

[51].Kato, M., N. E. Castro and R. Natarajan (2013). "MicroRNAs: potential mediators and biomarkers of diabetic complications." Free Radic Biol Med 64: 85-94.

[52]. Kdoqi (2007). "KDOQI Clinical Practice Guidelines and Clinical Practice Recommendations for Diabetes and Chronic Kidney Disease." Am J Kidney Dis 49(2 Suppl 2): S12-154.

[53]. Khella, H. W., M. Bakhet, Z. Lichner, A. D. Romaschin, M. A. Jewett and G. M. Yousef (2013). "MicroRNAs in kidney disease: an emerging understanding." Am J Kidney Dis 61(5): 798-808.

[54]. Kim, N. H., J. H. Oh, J. A. Seo, K. W. Lee, S. G. Kim, K. M. Choi, S. H. Baik, D. S. Choi, Y. S. Kang, S. Y. Han, K. H. Han, Y. H. Ji and D. R. Cha (2005). "Vascular endothelial growth factor (VEGF) and soluble VEGF receptor FLT-1 in diabetic nephropathy." Kidney Int 67(1): 167-177.

[55]. Kim, S. S., S. H. Song, I. J. Kim, W. J. Kim, Y. K. Jeon, B. H. Kim, I. S. Kwak, E. K. Lee and Y. K. Kim (2014). "Nonalbuminuric proteinuria as a biomarker for tubular damage in early development of nephropathy with type 2 diabetic patients." Diabetes Metab Res Rev.

[56]. Kim, S. S., S. H. Song, I. J. Kim, Y. K. Jeon, B. H. Kim, I. S. Kwak, E. K. Lee and Y. K. Kim (2013). "Urinary cystatin C and tubular proteinuria predict progression of diabetic nephropathy." Diabetes Care 36(3): 656-661.

[57]. Kitazawa, M., M. Nagano, K. H. Masumoto, Y. Shigeyoshi, T. Natsume and S. Hashimoto (2011). "Angiopoietin-like 2, a circadian gene, improves type 2 diabetes through potentiation of insulin sensitivity in mice adipocytes." Endocrinology 152(7): 2558-2567.

[58]. Korpinen, E., A. M. Teppo, L. Hukkanen, H. K. Akerblom, C. Gronhagen-Riska and O. Vaarala (2000). "Urinary transforming growth factor-beta1 and alpha1-microglobulin in children and adolescents with type 1 diabetes." Diabetes Care 23(5): 664-668.

[59]. Kotajima, N., T. Kimura, T. Kanda, K. Obata, A. Kuwabara, Y. Fukumura and I. Kobayashi (2000). "Type IV collagen as an early marker for diabetic nephropathy in non-insulin-dependent diabetes mellitus." J Diabetes Complications 14(1): 13-17.

[60]. Krupa, A., R. Jenkins, D. D. Luo, A. Lewis, A. Phillips and D. Fraser (2010). "Loss of MicroRNA-192 promotes fibrogenesis in diabetic nephropathy." J Am Soc Nephrol 21(3): 438-447.

[61]. Kuroda, A., T. A. Rauch, I. Todorov, H. T. Ku, I. H. Al-Abdullah, F. Kandeel, Y. Mullen, G. P. Pfeifer and K. Ferreri (2009). "Insulin gene expression is regulated by DNA methylation." PLoS One 4(9): e6953.

[62].Lan, H. Y. (2012). "Transforming growth factor-beta/Smad signalling in diabetic nephropathy." Clin Exp Pharmacol Physiol 39(8): 731-738.

[63].Lapsley, M., F. V. Flynn and P. A. Sansom (1993). "Beta 2-glycoprotein-1 (apolipoprotein H) excretion and renal tubular malfunction in diabetic patients without clinical proteinuria." J Clin Pathol 46(5): 465-469.

[64].Laterza, O. F., C. P. Price and M. G. Scott (2002). "Cystatin C: an improved estimator of glomerular filtration rate?" Clin Chem 48(5): 699-707.

[65].Lee, E. Y., S. S. Kim, J. S. Lee, I. J. Kim, S. H. Song, S. K. Cha, K. S. Park, J. S. Kang and C. H. Chung (2014). "Soluble alpha-klotho as a novel biomarker in the early stage of nephropathy in patients with type 2 diabetes." PLoS One 9(8): e102984.

[66].Lee, S. Y. and M. E. Choi (2014). "Urinary biomarkers for early diabetic nephropathy: beyond albuminuria." Pediatr Nephrol.

[67].Leinonen, J., T. Lehtimaki, S. Toyokuni, K. Okada, T. Tanaka, H. Hiai, H. Ochi, P. Laippala, V. Rantalaiho, O. Wirta, A. Pasternack and H. Alho (1997). "New biomarker evidence of oxidative DNA damage in patients with non-insulin-dependent diabetes mellitus." FEBS Lett 417(1): 150-152. 
[68]. Li, Q., W. Gong, Z. Yang, B. Lu, Y. Yang, W. Zhao and R. Hu (2013). "Serum Angptl2 levels are independently associated with albuminuria in type 2 diabetes." Diabetes Res Clin Pract 100(3): 385-390.

[69].Lin, C. L., P. H. Lee, Y. C. Hsu, C. C. Lei, J. Y. Ko, P. C. Chuang, Y. T. Huang, S. Y. Wang, S. L. Wu, Y. S. Chen, W. C. Chiang, J. Reiser and F. S. Wang (2014). "MicroRNA-29a promotion of nephrin acetylation ameliorates hyperglycemia-induced podocyte dysfunction." J Am Soc Nephrol 25(8): 1698-1709.

[70]. Mackinnon, B., L. Shakerdi, C. J. Deighan, J. G. Fox, D. S. O'Reilly and M. Boulton-Jones (2003). "Urinary transferrin, high molecular weight proteinuria and the progression of renal disease." Clin Nephrol 59(4): 252-258.

[71]. Matheson, A., M. D. Willcox, J. Flanagan and B. J. Walsh (2010). "Urinary biomarkers involved in type 2 diabetes: a review." Diabetes Metab Res Rev 26(3): 150-171.

[72]. McClelland, A. D. and P. Kantharidis (2014). "microRNA in the development of diabetic complications." Clin Sci (Lond) 126(2): 95-110.

[73]. McClelland, A., S. Hagiwara and P. Kantharidis (2014). "Where are we in diabetic nephropathy: microRNAs and biomarkers?" Curr Opin Nephrol Hypertens 23(1): 80-86.

[74]. McMahon, B. A. and P. T. Murray (2010). "Urinary liver fatty acid-binding protein: another novel biomarker of acute kidney injury." Kidney Int 77(8): 657-659.

[75]. Mojiminiyi, O. A. and N. Abdella (2003). "Evaluation of cystatin C and beta-2 microglobulin as markers of renal function in patients with type 2 diabetes mellitus." J Diabetes Complications 17(3): 160-168.

[76]. Moresco, R. N., M. B. Sangoi, J. A. De Carvalho, E. Tatsch and G. V. Bochi (2013). "Diabetic nephropathy: traditional to proteomic markers." Clin Chim Acta 421: 17-30.

[77]. Nakamura, T., C. Ushiyama, S. Suzuki, M. Hara, N. Shimada, I. Ebihara and H. Koide (2000). "Urinary excretion of podocytes in patients with diabetic nephropathy." Nephrol Dial Transplant 15(9): 1379-1383.

[78]. Nakamura, T., T. Sugaya, Y. Kawagoe, Y. Ueda, S. Osada and H. Koide (2005). "Effect of pitavastatin on urinary liver-type fatty acid-binding protein levels in patients with early diabetic nephropathy." Diabetes Care 28(11): 2728-2732.

[79]. Narita, T., H. Sasaki, M. Hosoba, T. Miura, N. Yoshioka, T. Morii, T. Shimotomai, J. Koshimura, H. Fujita, M. Kakei and S. Ito (2004). "Parallel increase in urinary excretion rates of immunoglobulin $\mathrm{G}$, ceruloplasmin, transferrin, and orosomucoid in normoalbuminuric type 2 diabetic patients." Diabetes Care 27(5): 1176-1181.

[80]. Narita, T., M. Hosoba, M. Kakei and S. Ito (2006). "Increased urinary excretions of immunoglobulin g, ceruloplasmin, and transferrin predict development of microalbuminuria in patients with type 2 diabetes." Diabetes Care 29(1): 142-144.

[81]. Nauta, F. L., W. E. Boertien, S. J. Bakker, H. van Goor, W. van Oeveren, P. E. de Jong, H. Bilo and R. T. Gansevoort (2011). "Glomerular and tubular damage markers are elevated in patients with diabetes." Diabetes Care 34(4): 975-981.

[82]. Navarro, J. F., C. Mora, M. Gomez, M. Muros, C. Lopez-Aguilar and J. Garcia (2008). "Influence of renal involvement on peripheral blood mononuclear cell expression behaviour of tumour necrosis factor-alpha and interleukin-6 in type 2 diabetic patients." Nephrol Dial Transplant 23(3): 919-926.

[83]. Navarro, J. F., C. Mora, M. Maca and J. Garca (2003). "Inflammatory parameters are independently associated with urinary albumin in type 2 diabetes mellitus." Am J Kidney Dis 42(1): 53-61.

[84]. Navarro, J. F., C. Mora, M. Muros and J. Garcia (2006). "Urinary tumour necrosis factor-alpha excretion independently correlates with clinical markers of glomerular and tubulointerstitial injury in type 2 diabetic patients." Nephrol Dial Transplant 21(12): 3428-3434.

[85]. Nguyen, T. Q., L. Tarnow, S. Andersen, P. Hovind, H. H. Parving, R. Goldschmeding and F. A. van Nieuwenhoven (2006). "Urinary connective tissue growth factor excretion correlates with clinical markers of renal disease in a large population of type 1 diabetic patients with diabetic 
nephropathy." Diabetes Care 29(1): 83-88.

[86]. Nielsen, S. E., H. Reinhard, D. Zdunek, G. Hess, O. M. Gutierrez, M. Wolf, H. H. Parving, P. K. Jacobsen and P. Rossing (2012). "Tubular markers are associated with decline in kidney function in proteinuric type 2 diabetic patients." Diabetes Res Clin Pract 97(1): 71-76.

[87]. Nielsen, S. E., K. J. Schjoedt, A. S. Astrup, L. Tarnow, M. Lajer, P. R. Hansen, H. H. Parving and P. Rossing (2010). "Neutrophil Gelatinase-Associated Lipocalin (NGAL) and Kidney Injury Molecule 1 (KIM1) in patients with diabetic nephropathy: a cross-sectional study and the effects of lisinopril." Diabet Med 27(10): 1144-1150.

[88]. Nielsen, S. E., T. Sugaya, L. Tarnow, M. Lajer, K. J. Schjoedt, A. S. Astrup, T. Baba, H. H. Parving and P. Rossing (2009). "Tubular and glomerular injury in diabetes and the impact of ACE inhibition." Diabetes Care 32(9): 1684-1688.

[89]. O'Donnell, M. J., J. Watson, P. Martin, C. Chapman and A. H. Barnett (1991). "Transferrinuria in type 2 diabetes: the effect of glycaemic control." Ann Clin Biochem 28 ( Pt 2): 174-178.

[90]. Ohara, N., O. Hanyu, S. Hirayama, O. Nakagawa, Y. Aizawa, S. Ito and H. Sone (2014). "Hypertension increases urinary excretion of immunoglobulin $\mathrm{G}$, ceruloplasmin and transferrin in normoalbuminuric patients with type 2 diabetes mellitus." J Hypertens 32(2): 432-438.

[91]. Oike, Y. and M. Tabata (2009). "Angiopoietin-like proteins--potential therapeutic targets for metabolic syndrome and cardiovascular disease." Circ J 73(12): 2192-2197.

[92]. Osipova, J., D. C. Fischer, S. Dangwal, I. Volkmann, C. Widera, K. Schwarz, J. M. Lorenzen, C. Schreiver, U. Jacoby, M. Heimhalt, T. Thum and D. Haffner (2014). "Diabetes-associated microRNAs in pediatric patients with type 1 diabetes mellitus: a cross-sectional cohort study." J Clin Endocrinol Metab 99(9): E1661-1665.

[93]. Ouchi, N., J. L. Parker, J. J. Lugus and K. Walsh (2011). "Adipokines in inflammation and metabolic disease." Nat Rev Immunol 11(2): 85-97.

[94]. Panduru, N. M., C. Forsblom, M. Saraheimo, L. Thorn, A. Bierhaus, P. M. Humpert, P. H. Groop and G. FinnDiane Study (2013). "Urinary liver-type fatty acid-binding protein and progression of diabetic nephropathy in type 1 diabetes." Diabetes Care 36(7): 2077-2083.

[95].Park, J. H., D. A. Stoffers, R. D. Nicholls and R. A. Simmons (2008). "Development of type 2 diabetes following intrauterine growth retardation in rats is associated with progressive epigenetic silencing of Pdx1." J Clin Invest 118(6): 2316-2324.

[96]. Patari, A., C. Forsblom, M. Havana, H. Taipale, P. H. Groop and H. Holthofer (2003). "Nephrinuria in diabetic nephropathy of type 1 diabetes." Diabetes 52(12): 2969-2974.

[97].Patnaik, S. K., R. Mallick and S. Yendamuri (2010). "Detection of microRNAs in dried serum blots." Anal Biochem 407(1): 147-149.

[98]. Perkins, B. A., R. G. Nelson, B. E. Ostrander, K. L. Blouch, A. S. Krolewski, B. D. Myers and J. H. Warram (2005). "Detection of renal function decline in patients with diabetes and normal or elevated GFR by serial measurements of serum cystatin C concentration: results of a 4-year follow-up study." J Am Soc Nephrol 16(5): 1404-1412.

[99]. Pirola, L., A. Balcerczyk, J. Okabe and A. El-Osta (2010). "Epigenetic phenomena linked to diabetic complications." Nat Rev Endocrinol 6(12): 665-675.

[100]. Piwowar, A., M. Knapik-Kordecka, I. Fus and M. Warwas (2006). "Urinary activities of cathepsin B, N-acetyl-beta-D-glucosaminidase, and albuminuria in patients with type 2 diabetes mellitus." Med Sci Monit 12(5): CR210-214.

[101]. Pucci, L., S. Triscornia, D. Lucchesi, C. Fotino, G. Pellegrini, E. Pardini, R. Miccoli, S. Del Prato and G. Penno (2007). "Cystatin C and estimates of renal function: searching for a better measure of kidney function in diabetic patients." Clin Chem 53(3): 480-488.

[102]. Qin, L. X., X. Zeng and G. Huang (2004). "[Changes in serum and urine ceruloplasmin concentrations in type 2 diabetes]." Zhong Nan Da Xue Xue Bao Yi Xue Ban 29(2): 208-211.

[103]. Salem, E. S., N. Grobe and K. M. Elased (2014). "Insulin treatment attenuates renal ADAM17 and ACE2 shedding in diabetic Akita mice." Am J Physiol Renal Physiol 306(6): F629-639.

[104]. Schena, F. P., G. Serino and F. Sallustio (2014). "MicroRNAs in kidney diseases: new 
promising biomarkers for diagnosis and monitoring." Nephrol Dial Transplant 29(4): 755-763.

[105]. Serdar, M., E. Sertoglu, M. Uyanik, S. Tapan, K. Akin, C. Bilgi and I. Kurt (2012). "Comparison of 8-hydroxy-2'-deoxyguanosine (8-OHdG) levels using mass spectrometer and urine albumin creatinine ratio as a predictor of development of diabetic nephropathy." Free Radic Res 46(10): 1291-1295.

[106]. Sharma, K., F. N. Ziyadeh, B. Alzahabi, T. A. McGowan, S. Kapoor, B. R. Kurnik, P. B. Kurnik and L. S. Weisberg (1997). "Increased renal production of transforming growth factor-beta1 in patients with type II diabetes." Diabetes 46(5): 854-859.

[107]. Shikano, M., H. Sobajima, H. Yoshikawa, T. Toba, H. Kushimoto, H. Katsumata, M. Tomita and S. Kawashima (2000). "Usefulness of a highly sensitive urinary and serum IL-6 assay in patients with diabetic nephropathy." Nephron 85(1): 81-85.

[108]. Shimizu, H., M. Negishi, Y. Shimomura and M. Mori (1992). "Changes in urinary retinol binding protein excretion and other indices of renal tubular damage in patients with non-insulin dependent diabetes." Diabetes Res Clin Pract 18(3): 207-210.

[109]. Srivastava, S. P., D. Koya and K. Kanasaki (2013). "MicroRNAs in kidney fibrosis and diabetic nephropathy: roles on EMT and EndMT." Biomed Res Int 2013: 125469.

[110]. Sthaneshwar, P. and S. P. Chan (2010). "Urinary type IV collagen levels in diabetes mellitus." Malays J Pathol 32(1): 43-47.

[111]. Sun, H., J. M. Zheng, S. Chen, C. H. Zeng, Z. H. Liu and L. S. Li (2007). "Enhanced expression of ANGPTL2 in the microvascular lesions of diabetic glomerulopathy." Nephron Exp Nephrol 105(4): e117-123.

[112]. Suzuki, K., T. Babazono, H. Murata and Y. Iwamoto (2005). "Clinical significance of urinary liver-type fatty acid-binding protein in patients with diabetic nephropathy." Diabetes Care 28(8): 2038-2039.

[113]. "Standards of medical care in diabetes--2007." Diabetes Care 30 Suppl 1: S4-S41.

[114]. Tabata, M., T. Kadomatsu, S. Fukuhara, K. Miyata, Y. Ito, M. Endo, T. Urano, H. J. Zhu, H. Tsukano, H. Tazume, K. Kaikita, K. Miyashita, T. Iwawaki, M. Shimabukuro, K. Sakaguchi, T. Ito, N. Nakagata, T. Yamada, H. Katagiri, M. Kasuga, Y. Ando, H. Ogawa, N. Mochizuki, H. Itoh, T. Suda and Y. Oike (2009). "Angiopoietin-like protein 2 promotes chronic adipose tissue inflammation and obesity-related systemic insulin resistance." Cell Metab 10(3): 178-188.

[115]. Tam, F. W., B. L. Riser, K. Meeran, J. Rambow, C. D. Pusey and A. H. Frankel (2009). "Urinary monocyte chemoattractant protein-1 (MCP-1) and connective tissue growth factor (CCN2) as prognostic markers for progression of diabetic nephropathy." Cytokine 47(1): 37-42.

[116]. Tan, Y., Y. Yang, Z. Zhang, X. Zhang, Z. Zhang and Y. Liu (2002). "Urinary type IV collagen: a specific indicator of incipient diabetic nephropathy." Chin Med J (Engl) 115(3): 389-394.

[117]. Tanaka, A., K. Shima, M. Fukuda, Y. Tahara, Y. Yamamoto and Y. Kumahara (1989). "Tubular dysfunction in the early stage of diabetic nephropathy." Med J Osaka Univ 38(1-4): 57-63.

[118]. Tashiro, K., I. Koyanagi, A. Saitoh, A. Shimizu, T. Shike, C. Ishiguro, M. Koizumi, K. Funabiki, S. Horikoshi, I. Shirato and Y. Tomino (2002). "Urinary levels of monocyte chemoattractant protein-1 (MCP-1) and interleukin-8 (IL-8), and renal injuries in patients with type 2 diabetic nephropathy." J Clin Lab Anal 16(1): 1-4.

[119]. Thrailkill, K. M., C. H. Jo, G. E. Cockrell, C. S. Moreau and J. L. Fowlkes (2011). "Enhanced excretion of vitamin D binding protein in type 1 diabetes: a role in vitamin D deficiency?" $\mathrm{J}$ Clin Endocrinol Metab 96(1): 142-149.

[120]. Tian, X. Q., L. M. Zhao, J. P. Ge, Y. Zhang and Y. C. Xu (2014). "Elevated urinary level of vitamin D-binding protein as a novel biomarker for diabetic nephropathy." Exp Ther Med 7(2): 411-416.

[121]. Timoshanko, J. R., J. D. Sedgwick, S. R. Holdsworth and P. G. Tipping (2003). "Intrinsic renal cells are the major source of tumor necrosis factor contributing to renal injury in murine crescentic glomerulonephritis." J Am Soc Nephrol 14(7): 1785-1793.

[122]. Titan, S. M., J. M. Vieira, Jr., W. V. Dominguez, S. R. Moreira, A. B. Pereira, R. T. Barros and R. Zatz (2012). "Urinary MCP-1 and RBP: independent predictors of renal outcome in 
macroalbuminuric diabetic nephropathy." J Diabetes Complications 26(6): 546-553.

[123]. Tofik, R., O. Torffvit, B. Rippe and O. Bakoush (2012). "Urine IgM-excretion as a prognostic marker for progression of type 2 diabetic nephropathy." Diabetes Res Clin Pract 95(1): 139-144.

[124]. Togashi, Y. and Y. Miyamoto (2013). "Urinary cystatin C as a biomarker for diabetic nephropathy and its immunohistochemical localization in kidney in Zucker diabetic fatty (ZDF) rats." Exp Toxicol Pathol 65(5): 615-622.

[125]. Viberti, G. C., H. Keen and D. Mackintosh (1981). "Beta 2-microglobulinaemia: a sensitive index of diminishing renal function in diabetics." Br Med J (Clin Res Ed) 282(6258): 95-98.

[126]. Villeneuve, L. M. and R. Natarajan (2010). "The role of epigenetics in the pathology of diabetic complications." Am J Physiol Renal Physiol 299(1): F14-25.

[127]. Wang, C., C. Li, W. Gong and T. Lou (2013). "New urinary biomarkers for diabetic kidney disease." Biomark Res 1(1): 9.

[128]. Weber, J. A., D. H. Baxter, S. Zhang, D. Y. Huang, K. H. Huang, M. J. Lee, D. J. Galas and K. Wang (2010). "The microRNA spectrum in 12 body fluids." Clin Chem 56(11): 1733-1741.

[129]. Weil, E. J., K. V. Lemley, B. Yee, T. Lovato, M. Richardson, B. D. Myers and R. G. Nelson (2011). "Podocyte detachment in type 2 diabetic nephropathy." Am J Nephrol 33 Suppl 1: 21-24.

[130]. Wu, C., Q. Wang, C. Lv, N. Qin, S. Lei, Q. Yuan and G. Wang (2014). "The changes of serum sKlotho and NGAL levels and their correlation in type 2 diabetes mellitus patients with different stages of urinary albumin." Diabetes Res Clin Pract.

[131].Zhang, Z., X. Luo, S. Ding, J. Chen, T. Chen, X. Chen, H. Zha, L. Yao, X. He and H. Peng (2012). "MicroRNA-451 regulates p38 MAPK signaling by targeting of Ywhaz and suppresses the mesangial hypertrophy in early diabetic nephropathy." FEBS Lett 586(1): 20-26.

[132].Zheng, M., L. L. Lv, J. Ni, H. F. Ni, Q. Li, K. L. Ma and B. C. Liu (2011). "Urinary podocyte-associated mRNA profile in various stages of diabetic nephropathy." PLoS One 6(5): e20431.

[133].Zhou, Y., X. Zhang and J. Wu (1997). "[Clinical significance of microtransferrinuria in diabetic patients]." Zhonghua Nei Ke Za Zhi 36(3): 165-168.

\section{AUTHOR's BIOGRAPHY}

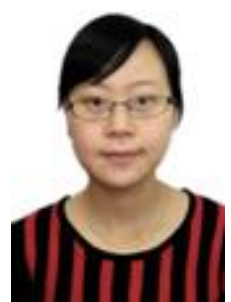

Lihua Liu, female,was born in March,1985. She graduated from the Jilin University in 2011 and got a master's degree,majoring in glomerular diseases and pathology.After graduating, She began to work in the Department of nephrology, the second hospital of Jilin University.She is a lecturer.She is good at diagnosis and treatment of common renal disease.Now She is a doctoral student in reading,major in the pathogenesis of diabetic nephropathy. 